\title{
Anatomic Stage IIIB Breast Cancer AJCC v8
}

National Cancer Institute

\section{Source}

National Cancer Institute. Anatomic Stage IIIB Breast Cancer A/CC v8. NCI Thesaurus.

Code 139543.

Stage IIIB includes: (T4, N0, M0); ( T4, N1, M0); (T4, N2, M0). T4: Tumor of any size with direct extension to the chest wall and/or to the skin (ulceration or macroscopic nodules); invasion of the dermis alone does not qualify as T4. N0: No regional lymph node metastasis is identified or isolated tumor cell clusters (ITCS) are identified only. N1: Tumor with micrometastases; or metastases in 1-3 axillary lymph nodes; and/or clinically negative internal mammary nodes with micrometastases or macrometastases by sentinel lymph node biopsy. N2: T umor with metastases in 4-9 axillary lymph nodes; or positive ipsilateral internal mammary lymph nodes by imaging in the absence of axillary lymph node metastases. M0: No clinical or radiog raphic evidence of distant metastases. Imaging studies are not required to assign the M0 category. (AJCC 8th ed.) 